\title{
Maximizing Lifetime of Heterogeneous Wireless Sensor Network using Heed Protocol
}

\author{
Tanmeet Kaur \\ Department of Electronics and Communication \\ CGC Group of Colleges \\ Mohali, India
}

\author{
Priyanka Sharma \\ Department of Electronics and Communication \\ CGC Group of Colleges \\ Mohali, India
}

\begin{abstract}
Wireless sensor network (WSN) has significant role in daily life. Many routing protocols have been proposed in recent advances, based on heterogeneity. Achieving the lifetime, deployment of nodes, fault tolerance , and energy efficiency are the main research goals .In this paper, we have proposed Hybrid Energy Efficient Distributed protocol (HEED) with heterogeneity to prolong the network lifetime. The significant improvement has been shown using HEED with heterogeneity in comparison with HEED in terms of energy consumption and data transmission to base station (B.S). The impact of heterogeneity of nodes on wireless sensor network(WSN) is studied and finally, the simulation results demonstrate that HEED with heterogeneity achieves longer lifetime and more better performance than HEED protocol.
\end{abstract}

\section{Keywords}

Wireless sensor network, Heterogeneity, Clustering, Network lifetime (NL)

\section{INTRODUCTION}

Wireless sensor network (WSN)has significant role in daily life. Application of sensor network involves defense, health, education, transportation, industries etc. wireless sensor network are randomly deployed in a field. Sensor network consist of number of nodes. A sensor node usually consist of four sub systems [1]. For each sensor node the energy source is battery, so in case the battery goes down, sensor node failed. Various protocols have been proposed to extend the life time of WSN. Network Life time is a key parameter which determine the performance of sensor network.

Network lifetime can be defined as time elapsed until the first node in the network deplete its energy. For example in a military field, where sensors are monitoring chemical activity, The life time of sensor is critical for maximum field coverage. Wasteful energy consumption can be due to idle listening to the media.

Hierarchical (Clustering) is the one of the method to reduce the energy consumption and improve life time. Clusters consist of number of nodes and in the entire group of clusters the node amongst highest energy act as cluster head and are in charge of co-coordinating with other nodes. Cluster Head $(\mathrm{CH})$ play a vital role in improving the life time of wireless sensor network. Clustering provides scalability to hundred or thousand of nodes. Clustering is efficient for large scale network and prolonging the network life time. Clusters are formed based upon geographical location and remaining residual energy. Cluster head act as monitor and having more responsibility than cluster members.

Although many protocols proposed minimize energy consumption on forwarding paths to increase energy efficiency, such protocols do not necessarily prolong network lifetime when certain nodes are "popular" i.e. present on most forwarding paths in the network. With clustering, a popular node is guaranteed to "lose its popularity" when new clusters are constructed.

In WSN sensor nodes are deployed in a field .Sensor nodes co-ordinate to produce high quality information about the physical environment .Sensor node bases its decisions to its target the information it already has and knowledge of its computing, communication. Each sensor node collect the data and route to base station. It is not necessary that all nodes are communicating at particular time..Base station may communicate with the Remote Controller node via internet or satellite [1,2].

Clustering protocols have been investigated in the context of routing protocols[3]. In this work we present a general distributed clustering approach that considers a hybrid of energy and communication cost. Based on this approach, HEED(Hybrid, Energy-Efficient, Distributed) protocol is the clustering protocol .HEED has four primary objectives[4] (i)prolonging network lifetime by distributing energy consumption(ii) terminating the clustering process within a constant number of iterations (iii)producing well-distributed cluster heads.Our clustering approach does not make assumptions about the distribution of nodes or about node capabilities.

\section{LITERATURE REVIEW AND RESEARCH ISSUES}

Application specific nature of WSN makes it different from wireless networks. There are different research challenges in WSN. Cost and other application specific issues affect the communication properties of the system. One of example is that in wireless sensor network radio communication is of low power and short range when compared to other wireless communication network. In WSN size,cost,power are fundamental constraints and play an important role.

Main Issues In Sensor node Deployement

1. Deployment to achieve minimal energy consumption.

2. Deployment of sensor nodes for a large scale WSN.

3. Repositioning of sensor nodes to repair coverage holes.

4. Sensor nodes deployment to maximize the coverage in the network.

\subsection{Low Energy Adaptive Clustering} Hierarchy (LEACH):

W.R. Heinzelman ,A.P. Chandrakasan and H. Balakrishnan [5] introduced Low Energy Clustering Hierarchy (LEACH) Protocol in the year 2000 . It is one of the most popular hierarchical routing algorithms for sensor network. LEACH 
randomly select few sensor nodes as cluster heads and rotates this role to distribute the energy load among the sensors in network. In this the idea is to form clusters of the sensor nodes based on the received signal strength and use local cluster heads as routers to sink. Number of nodes that communicate directly with base station has been reduced with help of LEACH protocol .In LEACH,the cluster heads compress data arriving from member nodes and send an aggregated packet to base station(B.S) in order to reduce the amount of information that must be transmitted to B.S. the cluster head in LEACH is randomly rotated in every round. In order to reduce the amount of information that must be transmitted to base station. The cluster head in LEACH is randomly rotated in every round. In order to reduce inter and intra cluster interference. LEACH uses TDMA,CDMA. Basically the operation of LEACH is done in two steps, the set up phase and the steady phase. In set up phase the nodes are organized in to clusters and cluster heads $(\mathrm{CH})$ are selected This decision is done by choosing a random number between 0 and 1.The node become a cluster head for the current round if number is less than the following threshold.

$$
\mathrm{T}(\mathrm{n})=\mathrm{p} /\{1-\mathrm{p}(\operatorname{rmod}(1 / \mathrm{p}))\} \quad \text { if } \mathrm{n}_{-} \mathrm{G}
$$

Here $p$ is desired percentage of cluster head. $G$ is the set of nodes that are involved in $\mathrm{CH}$ election. The data is transferred to base station in the steady state phase. The cluster head node, after receiving all the data from its member nodes, performs aggregation before sending it to base station. After some time period, set up phase is restarted and new CH's are selected. There are some drawbacks that are linked with this protocol such as single -hop routing is used in which each node transmit directly to cluster head and sink. In dynamic clustering the idea leads to extra overhead due to cluster head changes. LEACH protocol assumes that all nodes are having same amount of energy. It also assume that $\mathrm{CH}$ consume approximately same amount of energy. .It is mostly suited for monitor machinery diagnosis and fault detection. But if the cluster head is far away from base station then it require large amount of energy to transmit the data to base station Multihop-LEACH (M-LEACH) protocol solve this problem by simply changing the transmission mode between cluster heads and base station by using the other cluster heads as relay stations to send data to base station. In LEACH protocol the cluster heads are not uniformly distribute.It improve the energy efficiency of LEACH protocol. In LEACH-C each node send their current location information and residual energy level to the sink. The sink will calculate the average node energy and find out which node energy level is below the average.

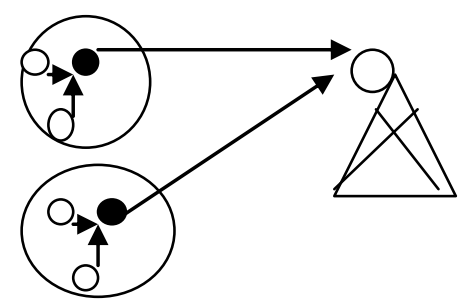

Fig: LEACH

\section{PEGASIS}

S.Lindsey and C.Raghavendra [6] proposed Power Efficient Gathering In Sensor Information Systems (PEGASIS) in year 2002.It is extension of LEACH protocol.This protocol is based on forming chains of sensor nodes, instead of forming
clusters.In this one node is responsible for routing aggregated data to sink,which act as cluster head.All node aggregates the collected data with its own data, and then forward the aggregated data to the next node. Only difference from LEACH is to employ multi hop transmission and choosing only one node to transmit to base station.The energy efficiency of PEGASIS is achieved by transmitting data to one of its neighbor node. The fused data will be forwarded to its one hop neighbor and collected data is fused.In this method each node will be getting the chance to forward gathered data to base station

\section{TEEN}

A.Manjeshwar and D.P. Agarwal [7] proposed Threshold Sensitive Energy Efficient Sensor Network Protocol (TEEN), protocol in year 2001.It works on idea that the closer nodes form clusters with the cluster heads to transmit the collected data to its one upper layer.In cluster's formation , cluster head's declare two threshold values. First one is hard threshold, and it is the minimum possible value of an attribute to trigger a sensor node. Second is the hard threshold, that allows nodes to transmit the event, if event occurs in the interested areas.Thus a significant reduction of transmission delay occurs .The nodes don't send a new data packet unless a change of minimum soft threshold occurs .Prevention from redundant data transmission is done by employing soft threshold .For time critical applications the protocol is very much suitable.

A.Manjeshwar and D.P. Agarwal [8] proposed Adaptive Threshold Sensitive Energy Efficient Sensor Network Protocol in 2002. This protocol is an improvement of TEEN protocol. The aim of this protocol is to capture both time critical events and periodic data collections. The network design is same as TEEN.Data aggregation are also responsible is also formed by cluster head in order to decrease the size of data transmitted.So energy is consumed in this work.TEEN is better than LEACH and APTEEN because of the decreased number of transmissions. The main disadvantage of TEEN and APTEEN are overhead,to enforce the threshold based functions .The major disadvantage is the overhead and complexity of forming clusters in multiple levels.

\section{SEP}

G. Smaragdakis, I. Matta and A. Bestavros[9] introduced Stable Election Protocol (SEP) Protocol in year 2004. This protocol is aware of heterogeneity, based on weighted election probabilities of every node ready to become head of cluster by means of their respective energy.It is ensured in this approach that the election of cluster head is randomly selected and distributed based on the fraction of energy of each node guaranteeing a uniform use of nodes energy.Two type of nodes are considered in this protocol.

\section{EECS}

M. Ye, C. Li, G. Chen and J. Wu [10] proposed Energy Efficient Clustering Scheme (EECS) protocol in year 2005. For wireless sensor networks, it is novel clustering scheme for periodical data gathering applications. The nodes with more residual energy elects as cluster heads through local radio communication. A constant number of candidate nodes are elected and compete for cluster heads as per to the node residual energy, in the cluster head election phase. In this localized competition is performed without iteration. In this method a near distribution of cluster heads are also produced. A novel approach is introduced to balance the load among 
cluster heads, in the cluster formation phase. The requirement of global knowledge about the distance between the clusterheads and base station is also increased in this.

\section{DEEC}

Q.Li, Z.Qingxin and W. Mingwen [11] proposed (DEEC) protocol in year 2006. This is a cluster based scheme protocol for multi level and two level energy heterogeneous wireless sensor networks. The cluster heads are selected by using the probability based on the ratio between residual energy of each node and the average energy of network, in this scheme. According to initial and residual energy of each node, the epochs of being cluster heads for nodes are different. The probability of becoming cluster heads is more based on nodes with high initial and residual energy compared to nodes with low energy.

\section{HEED}

O. Younis and S. Fahmy [4] proposed Hybrid Energy Efficient Distributed Clustering Protocol (HEED) protocol in year 2004. It is the extension of LEACH protocol by using residual energy as primary parameter and network topology features such as node degree, distances to neighbors are only used as secondary parameters to break tie between candidate cluster heads,for cluster selection to achieve power balancing. It divide clustering process in to number of iterations, nodes which are not covered by any cluster head double their probability of becoming a cluster head, in each iterations. Every node independently and probabilistically decides on its role in the clustered network in energy efficient clustering protocol. They cannot guarantee optimal elected set of cluster heads.

\section{FORMATION OF CLUSTER IN HEED PROTOCOL}

Network model is described in this section. Assume that in $100 \mathrm{~m} * 100 \mathrm{~m}$ square region $\mathrm{N}$ sensor nodes, are randomly dispersed. In this regarding the network model following assumptions are made:Nodes are left unattended after deployment.

1. Nodes in the network are quasi-stationary.

2. Nodes have similar processing and communication capabilities and equal significance.

3. Nodes location are unaware i.e it is not equipped by GPS capable antenna.

4. In terms of energy sensor nodes have heterogeneity i.e energy levels are different. All nodes have different initial energy . There are some nodes that are equipped with more energy than normal nodes.

In this selection of cluster head is primarily based on the residual energy of each node. In this residual energy can be estimated by knowing the energy consumed per bit for sensing, processing and communication. In this to break the ties intra cluster communication cost is considered as secondary parameter. In this tie means that a node fall with in the range of more than one cluster head.

Always the cluster head yielding lower intra cluster communication cost are favored When there are multiple candidate cluster heads. In this intra-cluster communication cost is considered as a secondary parameter. Intra cluster communication cost is a function of Whether or not variable power levels used are permissible for the intra-cluster communication Cluster properties, such as cluster size. The cost can be proportional to node degree and $1 /$ Node degree if the power level used for intra- cluster communication fixed for all nodes.

\section{- $\quad$ Node Degree :-}

If the main requirement is to distribute load among cluster heads.

\section{- $\quad 1 /$ Node Degree :-}

If to create dense clusters is requirement. Neighbor discovery is performed by each node And broadcast its cost to the detected neighbors. Before, a node start to executing HEED then each node set its probability of becoming a cluster head.

$\mathrm{CH}$ Prob $=\max \left(\mathrm{Cprob}^{*}(\right.$ Eresidual/Emax $\left.), \mathrm{pmin}\right)$

\section{WSN MODEL OF HETEROGENEITY}

In this paper, we describe the network model. Assume that there are $\mathrm{N}$ sensor nodes, which are dispersed uniformly with in a $\mathrm{M} * \mathrm{M}$ square region. The network consist of clustering hierarchy and data aggregation is executed by the cluster head in order to reduce the data that is redundant and produced by sensor nodes within the cluster. In heterogeneous WSN Model we consider the concept of heterogeneity.

\subsection{Two level HEED with heterogeneity}

In two level HEED with heterogeneity, two types of sensor nodes, i.e the advanced nodes and other are normal nodes. $\mathrm{E}_{0}$ is the initial energy of the normal nodes and $m$ is the percentage of advanced nodes, Which are having more energy than normal nodes by amount. Thus there are $\mathrm{m}^{*} \mathrm{~N}$ advanced nodes equipped with initial energy of $\mathrm{E}_{\mathrm{o}} *(1+\mathrm{a})$ and $(1-\mathrm{m}) * \mathrm{~N}$ normal nodes equipped with initial energy of $E_{0}$. The total initial energy of network [9] given by:

$$
\begin{aligned}
& \mathrm{E}_{\text {total }} \mathrm{N} *(1-\mathrm{m}) * \mathrm{E}_{0}+\mathrm{N} * \mathrm{~m} * \mathrm{E}_{0} *(1+\mathrm{a}) \\
& =\mathrm{N} * \mathrm{E}_{0} *(1+\mathrm{am})
\end{aligned}
$$

So, this type of networks has am more nodes and has am times more energy.

\subsection{Three level HEED with heterogeneity}

In three level HEED with heterogeneity protocol, there are three types of sensor nodes, i.e. the advanced nodes super nodes and normal nodes . Let $\mathrm{m}$ be the fraction of the total number of nodes $\mathrm{N}^{*} \mathrm{~m}$ which are equipped with $\beta$ times more energy than that of normal nodes which are called as super nodes. The number is $\mathrm{N}^{*} \mathrm{~m} * \mathrm{~m}_{0}$. The rest nodes i.e $\mathrm{N} * \mathrm{~m} *(1-$ $\mathrm{m}_{0}$ ) are the advanced nodes having a times more energy than normal nodes. The energy of each advanced node is $\mathrm{E}_{0} *(1+\mathrm{a})$ and the energy of each super node is $\mathrm{E}_{0} *(1+\beta)$

The total energy of the networks is as:

$\mathrm{E}_{\text {total }} \mathrm{N} *(1-\mathrm{m}) * \mathrm{E}_{0}+\mathrm{N} * \mathrm{~m} *\left(1-\mathrm{m}_{0}\right) * \mathrm{E}_{0} *$

$(1+a)+N * m * m_{0}+E_{0} *(1+\beta)$

$\mathrm{E}_{\text {total }}=\mathrm{N} * \mathrm{E}_{0} *\left(1+\mathrm{m} *\left(\mathrm{a}+\mathrm{m}_{0} * \beta\right)\right)$ 
So, the total energy of network is increased by the factor of $\left(1+m^{*}\left(a+m_{0} * \beta\right)\right)$

\section{Energy Model}

We used wireless radio dissipation model as proposed in [12] .The radio model in this has three modules i.e the power amplifier, the transmitter and the receiver. In energy model the transmitter dissipates energy to run the radio electronics and power amplifier.

The propagation loss of transmitting power is modeled as inversely proportional to $\mathrm{d}^{2}$, in the free space propagation model, where $\mathrm{d}$ is the distance between the transmitter and the receiver. The propagation loss of transmitting power is modeled as inversely proportional to $\mathrm{d}^{4}$. The energy dissipation for transmitting an $\mathrm{L}$ bit message from transmitter to receiver is defined as:

$E_{T x}(I, d)=\left\{\begin{array}{l}\text { I Eelec }+I_{E_{f s}} d^{2}, d<d_{0} \\ \text { I Eelec }+I_{m p} d^{4}, d \geq d_{0}\end{array}\right.$

\section{CONCLUSION}

In this paper, HEED with heterogeneity protocol for heterogeneous wireless sensor network is proposed. In this different levels of heterogeneity i.e two-level and three-level in term of node energy is introduced. We have evaluated the performance of proposed HEED with heterogeneity using Matlab. It is observed that there is significant improvement in the lifetime of HEED with heterogeneity in comparison with HEED protocol, because the number of iterations is maximum in heterogeneity concept.

\section{REFERENCES}

[1] V. Raghunathan, C. Schurgers, Park. S, and M. B.Srivastava. 2002. Energy-aware wireless micro sensor networks. IEEE Signal Processing Magazine, Volume: 19 Issue: 2, Page(s): $40-50$.

[2] I. Akyildiz et al. 2002. A Survey on Sensor Networks. IEEE Commun. Mag., vol. 40, no. 8, pp. 102-14.
[3] v.kawadia and P.R. kumar "power control and clustering in Ad HOC Networks". In proceeding of IEEE INFOCOM, April 2003.

[4] Ossama Younis and Sonia Fahmy. 2004. Distributed Clustering in Ad-hoc Sensor Networks: A Hybrid, Energy-Efficient Approach. In Proceedings of IEEE INFOCOM, Hong Kong, an extended version appeared in IEEE Transactions on Mobile Computing, 3(4).

[5] W. Heinzelman, A. Chandrakasan and H. Balakrishnan.2000. Energy-Efficient Communication Protocol for Wireless Microsensor Networks. Proceedings of the $33^{\text {rd }}$ Hawaii International Conference on System Sciences(HICSS '00).

[6] S. Lindsey, C. Raghavendra. 2002. PEGASIS: PowerEfficient Gathering in Sensor Information Systems. IEEE Aerospace Conference Proceedings, Vol. 3, 9-16 pp. $1125-1130$

[7] A. Manjeshwar and D. P. Agarwal. 2001. TEEN: a routing protocol for enhanced efficiency in wireless sensor networks. In 1st International Workshop on Parallel and Distributed Computing Issues in Wireless Networks and Mobile Computing.

[9] G. Smaragdakis, I. Matta, A. Bestavros. 2004. SEP: A Stable Election Protocol for clustered heterogeneous wireless sensor networks. In Second International Workshop on Sensor and Actor Network Protocols and Applications (SANPA).

[10] M. Ye, C. Li, G. Chen, J. Wu. 2005. EECS: an energy efficient cluster scheme in wireless sensor networks. In IEEE International Workshop on Strategies for Energy Efficiency in Ad Hoc and Sensor Networks (IEEE IWSEEASN-2005), Phoenix, Arizona, April 7-9.

[11] Q. Li, Z. Qingxin, and W. Mingwen. 2006. Design of a distributed energy efficient clustering algorithm for heterogeneous wireless sensor networks. Computer Communications, vol. 29, pp. 2230-7.

[12] W.R. Heinzelman, A.P. Chandrakasan, H. Balakrishnan. 2002. An application-specific protocol architecture for wireless microsensor networks. IEEE Transactions on Wireless Communications 1 (4) 660-670. 